\title{
Evidence that memantine reduces chronic tinnitus caused by acoustic trauma in rats
}

\section{Yiwen Zheng, Emily McNamara, Lucy Stiles, Cynthia L. Darlington and Paul F. Smith*}

Department of Pharmacology and Toxicology, Brain Health Research Centre, School of Medical Sciences, University of Otago, Dunedin, New Zealand

\section{Edited by:}

G. M. Halmagyi, Royal Prince Alfred Hospital, Australia

\section{Reviewed by:}

Aasef G. Shaikh, Case Western

Reserve University, USA

Seong-Hae Jeong, Chungnam

National University Hospital, South

Korea

\section{${ }^{*}$ Correspondence}

Paul F. Smith, Department of

Pharmacology and Toxicology, Brain Health Research Centre, School of

Medical Sciences, University of

Otago, P.O. Box 913 Dunedin, New

Zealand.

e-mail:paul.smith@

stonebow.otago.ac.nz
Subjective tinnitus is a chronic neurological disorder in which phantom sounds are perceived. Increasing evidence suggests that tinnitus is caused by neuronal hyperactivity in auditory brain regions, either due to a decrease in synaptic inhibition or an increase in synaptic excitation. One drug investigated for the treatment of tinnitus has been the uncompetitive $\mathrm{N}$-methyl-D-aspartate (NMDA) receptor antagonist, memantine, although the evidence relating to it has been unconvincing to date. We re-investigated the effects of memantine on the behavioral manifestations of tinnitus induced by acoustic trauma (a $16-\mathrm{kHz}, 110-\mathrm{dB}$ pure tone presented unilaterally for $1 \mathrm{~h}$ ) in rats. We used a conditioned lick suppression model in which lick suppression was associated with the perception of high frequency sound resembling tinnitus and a suppression ratio (SR) was calculated by comparing the number of licks in the 15-s period preceding the stimulus presentation $(A)$ and the 15-s period during the stimulus presentation (B), i.e., $S R=B /(A+B)$. Acoustic trauma resulted in a significant increase in the auditory brainstem-evoked response (ABR) threshold in the affected ear $(P \leq 0.0001)$ and a decrease in the SR compared to sham controls in response to $32 \mathrm{kHz}$ tones in five out of eight acoustic trauma-exposed animals. A 5-mg/kg dose of memantine significantly reduced the proportion of these animals which exhibited tinnitus-like behavior ( $2 / 5$ compared to $5 / 5 ; P \leq 0.006)$, suggesting that the drug reduced tinnitus. These results suggest that memantine may reduce tinnitus caused by acoustic trauma.

Keywords: acoustic trauma, tinnitus, memantine, NMDA receptors, rats

\section{INTRODUCTION}

Subjective tinnitus has been estimated to occur in $25.3 \%$ of people in the USA, with $7.9 \%$ experiencing it frequently (Shargorodsky et al., 2010). Among other options, drugs are one of the main treatment avenues for severe tinnitus. However, despite the number of different drugs that are sometimes used for the condition, including benzodiazepines, anti-epileptic drugs, anti-spastic drugs, and even herbal medicines, there is surprisingly little convincing evidence to support their efficacy in consistently alleviating the condition (see Darlington and Smith, 2007; Elgoyhen and Langguth, 2011 for reviews).

Data from animal and human studies have suggested that tinnitus is associated with neuronal hyperactivity at different levels of the central auditory pathways, including the dorsal cochlear nucleus, the inferior colliculus, auditory cortex, and the striatum (see Moller, 2000; Eggermont and Roberts, 2004; Eggermont, 2005; Kaltenbach, 2006; Roberts et al., 2010 for reviews; see Dong et al., 2010; Rauschecker et al., 2010; Middleton et al., 2011; Mulders and Robertson, 2011; Vogler et al., 2011; Leaver et al., 2011 for recent examples). For this reason, drugs that increase synaptic inhibition, such as benzodiazepines and $\mathrm{GABA}_{\mathrm{B}}$ receptor agonists, have been one avenue of investigation for potential new

Abbreviations: SR, suppression ratio; ABR, auditory brainstem-evoked response; SPL, sound pressure level; BBN, broad band noise. treatments for tinnitus (Middleton et al., 2011; Wang et al., 2011; Zheng et al., 2012; see Darlington and Smith, 2007; Elgoyhen and Langguth, 2011 for reviews). However, the mechanisms underlying the neuronal hyperactivity associated with tinnitus are not entirely understood, and drugs that block excitatory synaptic neurotransmission may also be effective. For example, there is some evidence that salicylate-induced tinnitus may involve an increase in glutamatergic neurotransmission at the $\mathrm{N}$-methyl-D-aspartate (NMDA) subtype of glutamate receptor in the cochlea (Lin, 1997; Guitton et al., 2003) and that NMDA receptor antagonists can block this effect (Guitton et al., 2003). Moreover, long-term tinnitus induced by acoustic trauma was prevented by locally applying another NMDA receptor, polyamine site, antagonist, ifenprodil, into the cochlea within the first 4 days after the acoustic trauma (Guitton and Dudai, 2007).

Memantine is an uncompetitive antagonist at the NMDA receptor, which has been investigated as a potential treatment for tinnitus. Although it has a similar site of action in the NMDA receptor calcium channel to dizocilpine maleate (MK801), its different channel kinetics confer upon it a more favorable adverse side effect profile (Olivares et al., 2012). However, limited studies of the effects of memantine on tinnitus have shown complex results. Lobarinas et al. (2006) examined its effects on salicylate- and quinine-induced tinnitus in rats and found that it failed to reduce the behavioral indices of tinnitus and the increase in cortical auditory-evoked potentials caused by salicylate 
(see also Lobarinas et al., 2008). In what appears to be the only published clinical trial, Figueiredo et al. (2008) studied the effects of memantine in 60 patients with tinnitus. This was a randomized, double-blind, placebo-controlled cross-over trial in which tinnitus severity was quantified using the Tinnitus Handicap Inventory (THI). However, $20 \mathrm{mg}$ memantine per day for 90 days had no significant effect on the patients' tinnitus compared to placebo. More recently, Suckfull et al. (2011) have reported some beneficial effect in tinnitus patients of neramexane, which is also an NMDA receptor antagonist. However, it also acts at the $\alpha_{9} \alpha_{10}$ nicotinic acetylcholine and $5-\mathrm{HT}_{3}$ receptors and it is not clear which of its various actions might be responsible for its effects on tinnitus (Suckfull et al., 2011).

Given the small amount of evidence relating to memantine in the context of tinnitus and the fact that the drug is currently approved for the treatment of moderate to severe Alzheimer's disease (Olivares et al., 2012) and is therefore available clinically, we considered it necessary to re-investigate the potential of memantine to treat tinnitus, using an acoustic trauma animal model in which tinnitus was indicated by a reduced suppression ratio (SR) in a conditioned lick suppression paradigm (Zheng et al., 2011a,b,c, 2012).

\section{MATERIALS AND METHODS SUBJECTS}

Data were obtained from 16 male Wistar rats $(300-350 \mathrm{~g}$ at the beginning of the study) divided into acoustic trauma $(n=8)$ and sham control $(n=8)$ groups. The animals were maintained on a 12:12 h light:dark cycle at $22^{\circ} \mathrm{C}$ and were water deprived throughout the tinnitus behavioral tests. All procedures were approved by the University of Otago Committee on Ethics in the Care and Use of Laboratory Animals.

\section{DRUG ADMINISTRATION}

Memantine (Sigma) was dissolved in saline and animals received a daily $5 \mathrm{mg} / \mathrm{kg}$ s.c. injection $1 \mathrm{~h}$ before being tested with the conditioned lick suppression paradigm (see below), using a counterbalanced design. This dose of memantine was based on our own pilot experiments. Animals were first tested in the paradigm at 2 weeks following the acoustic trauma to confirm the presence of tinnitus and then tested following the vehicle (saline), $5 \mathrm{mg} / \mathrm{kg}$ memantine as well as during the washout of the drug. Each phase (pre-drug, vehicle, $5 \mathrm{mg} / \mathrm{kg}$ memantine, and washout) took 18 days of testing. Note that the counterbalanced design meant that noise trauma and sham animals received the drug and vehicle treatments in different orders and that the condition in which the noise trauma animals received the drug vehicle served as a control group for the condition in which the noise trauma animals received the drug.

\section{NOISE TRAUMA TO INDUCE CHRONIC TINNITUS IN RATS}

Unilateral acoustic trauma was delivered using a procedure described previously (Bauer and Brozoski, 2001; Brozoski et al., 2007; Zheng et al., 2011a,b,c, 2012). Briefly, the animals were anesthetized with ketamine $\mathrm{HCl}(75 \mathrm{mg} / \mathrm{kg}$, s.c.) and medetomidine hydrochloride $(0.3 \mathrm{mg} / \mathrm{kg}$, s.c.) and were placed inside a sound attenuation chamber for a $1 \mathrm{~h}$ exposure to a $16 \mathrm{kHz}, 110 \mathrm{~dB}$ sound pressure level (SPL) pure tone delivered to one of the ears as described in detail previously (Zheng et al., 2011a,b,c, 2012).

A $16 \mathrm{kHz}, 110 \mathrm{~dB}$ pure tone generated by a NI 4461 Dynamic Signal Acquisition and Generation system (National Instruments New Zealand Ltd.) was delivered to one of the ears through a closed field magnetic speaker with a tapered tip (Tucker-Davis Technologies), attached to a 3-mm cone-shaped speculum that was fitted tightly into the external auditory canal, for $1 \mathrm{~h}$. This kind of stimulus has previously been reported to induce tinnitus (Tan et al., 2007; Zheng et al., 2011a, 2012). Acoustic values were calibrated before noise exposure by connecting the speaker to a $1 / 4$-inch prepolarized free-field microphone (Type 40BE, GRAS Sound, and Vibration) via the speculum used to fit into the external auditory canal. The unexposed ear was blocked with cone-shaped foam and taped against the foam surface. The sham animals were kept under anesthesia for the same duration as the noise trauma animals, but without noise exposure.

\section{AUDITORY FUNCTION}

Auditory function in both ears of exposed and sham animals before and immediately after the acoustic trauma or sham treatment was measured using auditory brainstem-evoked response (ABR) thresholds in response to pure tones at 8,16 , and $20 \mathrm{kHz}$ (Zheng et al., 2011a,b,c, 2012). Briefly, the animals were anesthetized as previously described and subdermal needle electrodes were placed at the vertex and over the bullae with a reference electrode at the occiput. ABR thresholds were tested for tone bursts ( $2 \mathrm{~ms}$ rise/decay, $1 \mathrm{~ms}$ plateau) presented at a rate of $50 / \mathrm{s}$, in a decreasing intensity series, beginning with levels that elicited distinct evoked potentials. Hearing threshold was indicated by the lowest intensity that produced visually distinct potentials.

\section{TINNITUS ASSESSMENT}

The presence of tinnitus was assessed in each rat after the acoustic trauma using a conditioned lick suppression paradigm described in our previous publications (Zheng et al., 2011a,b,c, 2012). Tinnitus assessment was conducted in an operant conditioning test chamber (ENV-007, Med Associates Inc.) using a conditioned lick suppression paradigm, which we developed based on the lever pressing paradigm described by Brozoski et al. (2007). Drinking activity was measured by a lickometer with a photobeam (ENV-251L, Med Associates Inc.). A speaker (ENV-224DM, Med Associates Inc.) directly above the drinking tube produced broad band noise $(\mathrm{BBN})$ or a pure tone of different frequencies and intensities via a sound generator (ANL926, Med Associates Inc.). The stimulus intensities used were $0,30,40,50,70,80,90$, and $100 \mathrm{~dB}$ SPL. The BBN was white noise ranging from 3 to $20 \mathrm{kHz}$ with no equalization and the level was measured using an NI 4461 Dynamic Signal Acquisition and Generation card (National Instruments New Zealand Ltd.) and was calibrated as dB (SPL). The chamber floor was lined with stainless steel rods $(0.48 \mathrm{~cm}$ in diameter, ENV-005, Med Associates Inc.) and delivered an electric shock $(0.35 \mathrm{~mA})$ produced by a constant current shock source (ENV-410B, Med Associates Inc.) through a scrambler (ENV-412, Med Associates Inc.).

The conditioned lick suppression paradigm consisted of $15 \mathrm{~min}$ of testing every day and the animals went through three phases: the 
acclimation phase, the Pavlovian conditioned suppression training phase, and the frequency discrimination phase. During the acclimation phase, the BBN was played throughout the $15 \mathrm{~min}$ session except at 10 random intervals, at which point $15 \mathrm{~s}$ acoustic stimulus presentations were inserted. Two of the 10 presentations were always speaker off periods (i.e., silence) and the remaining eight were one of $\mathrm{BBN}, 10$ or $20 \mathrm{kHz}$ tones at one of four different intensity levels in a random order with each stimulus repeated twice within each session. The type of stimulus varied randomly between sessions, but remained constant within a session. We found that animals exposed to the acoustic trauma did not produce significantly more lick suppression than the sham animals to the $20 \mathrm{kHz}$ tones as we have observed in previous studies (Zheng et al., 2011a,b,c, 2012); therefore, we tested them at higher frequencies, including $32 \mathrm{kHz}$. We found that the noise-exposed rats did show the expected downward shift in the discrimination function at $32 \mathrm{kHz}$ compared with the sham rats, and therefore we substituted this higher frequency in the paradigm described above.

Following acclimation, each animal received conditioned suppression training in which a 3 -s foot shock $(0.35 \mathrm{~mA})$ was presented at the end of each speaker off period. The foot shock acted as an unconditioned stimulus (UCS) and the speaker off period acted as a conditioned stimulus (CS). Over a few sessions, the rats reacted to the speaker off by stopping licking (i.e., the conditioned suppression). The lick suppression was measured by comparing the number of licks in the 15-s period preceding the stimulus presentation (A) and the 15-s period during the stimulus presentation (B), i.e., the SR:

$$
\mathrm{SR}=\frac{\mathrm{B}}{\mathrm{A}+\mathrm{B}}
$$

Once the lick suppression was established ( $\mathrm{SR}<0.2)$, the rats were subjected to the frequency discrimination test, during which the acoustic stimuli were presented in the same way as in the acclimation and the suppression training (i.e., BBN or pure tones of 10,20 , or $32 \mathrm{kHz}$ at $0,30,40,50,70,80,90$, or $100 \mathrm{~dB}$ SPL). However, the foot shock was delivered only if the SR for the speaker off period was $>0.2$.

If a rat did not have tinnitus, the presentation of the stimuli had no effect on its drinking activity. However, if a rat had tinnitus, the tinnitus served as the CS during the training sessions, therefore, a stimulus with sensory features resembling tinnitus during the testing session would produce greater suppression. Each stimulus was tested five to six times. Tinnitus was first assessed in these rats at 2 weeks after the noise exposure. Although it was possible that the severity of tinnitus varied over the testing period, we controlled for this by counterbalancing the order of the treatment conditions.

\section{STATISTICAL ANALYSIS}

All data were first tested for the normal parametric assumptions of normality and homogeneity of variance (Kutner et al., 2005). Data for the ABRs and the pre-drug condition were then analyzed using a Linear Mixed Model (LMM) analysis using a restricted maximum likelihood procedure in SPSS 19 (Kutner et al., 2005; Gurka and Edwards, 2011). LMM analyses were used as an alternative to repeated measures ANOVAs because there was extensive autocorrelation in the data; LMM analyses model the covariance structure of the repeated measures data in order to address this problem (Kutner et al., 2005; Norusis, 2010; Gurka and Edwards, 2011). The most appropriate covariance matrix structure was chosen based on the smallest Akaike's Information Criterion (Norusis, 2010). Group (noise trauma versus sham), stimulus intensity, and the interaction between group and stimulus intensity were evaluated. While a significant group effect indicated significant differences between the noise trauma and sham groups irrespective of stimulus intensity, a significant interaction between group and intensity indicated a significant difference between groups as a function of intensity. $P<0.05$ was considered significant.

We recognized that not all animals exposed to acoustic trauma necessarily develop tinnitus and that therefore the inclusion of animals that did not have tinnitus or had only mild tinnitus, could bias the results of the drug study (Heffner and Harrington, 2002). For this reason, we also analyzed the data for individual animals both pre-drug and following drug treatment, using the criterion that if three or more mean SR values for an individual animal were less than the mean for the sham controls, this animal could be considered to exhibit evidence of tinnitus. We then used a test for two proportions to determine whether the same animals which exhibited tinnitus in the pre-drug condition, also exhibited tinnitus in the memantine and washout conditions (Agresti, 2007; Sprent and Smeeton, 2007).

\section{RESULTS AUDITORY FUNCTION AND THE CONFIRMATION OF TINNITUS}

Unilateral acoustic trauma produced an immediate loss of auditory function in the exposed ear at 16 and $20 \mathrm{kHz}$ as indicated by the elevated ABR thresholds $[F(1,167)=16.52, P \leq 0.0001$ for group and $F(1,167)=23.74$ for the group $\times$ ear $\times$ time interaction, $P \leq 0.0001$, i.e., the affected ear in the noise-exposed group showed a significant change in the ABR threshold between the pre- and post-exposure measurements; Figure 1]. However, in this group of animals the ABR thresholds were not significantly different from sham controls at $8 \mathrm{kHz}$ (Figure 1). Auditory function in the ears of sham-exposed animals and in the unexposed ears of the acoustic trauma-exposed animals, were not affected. Although we did not test ABR thresholds in these animals at the end of this experiment, in a similar experiment involving eight sham and eight acoustic trauma-exposed rats, and exactly the same methods, we found no significant differences in hearing threshold at 3 months post-exposure, despite a large and significant elevation of threshold in the ipsilateral, exposed ear immediately after the noise trauma (Figure 2; Zheng et al., unpublished observations).

In the conditioned suppression task, as expected, stimulus intensity always had a significant effect on the SR, i.e., SR increased with the increase in stimulus intensity for all the frequencies tested, and therefore will not be discussed further. When tested under the lick suppression paradigms at 2 weeks following the acoustic trauma and before any vehicle or drug treatment, there was a significant downward shift in the SR curve for the acoustic trauma group compared to the sham controls in response to the $32 \mathrm{kHz}$ tones [significant interaction between group and intensity: $F(4$, $285.09)=2.97, P \leq 0.02$ ] but not in response to the $\mathrm{BBN}$ or $10 \mathrm{kHz}$ tones (for either group or the interaction between group and intensity; data not shown), indicating the presence of tinnitus similar 


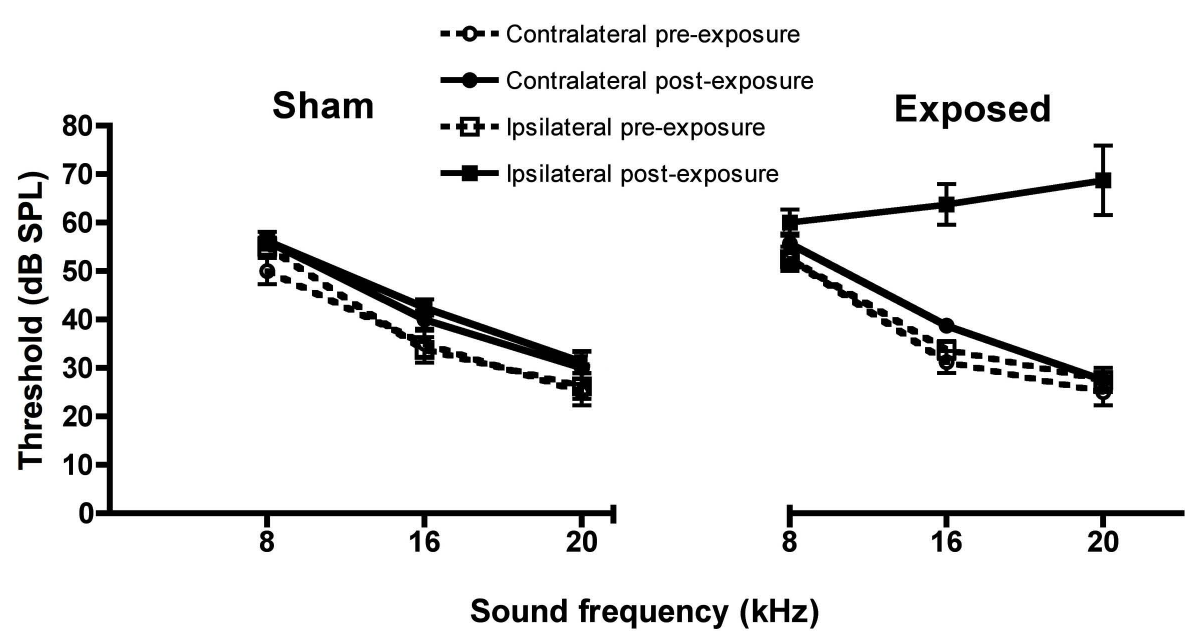

FIGURE 1 | Auditory brainstem-evoked response thresholds for the ipsilateral and contralateral ears of acoustic trauma-exposed and sham control animals before and after exposure, as a function of intensity in $\mathbf{~ B B ~ S P L}$ and frequency in $\mathbf{k H z}$. Bars represent means $\pm 1 \mathrm{SE}$.

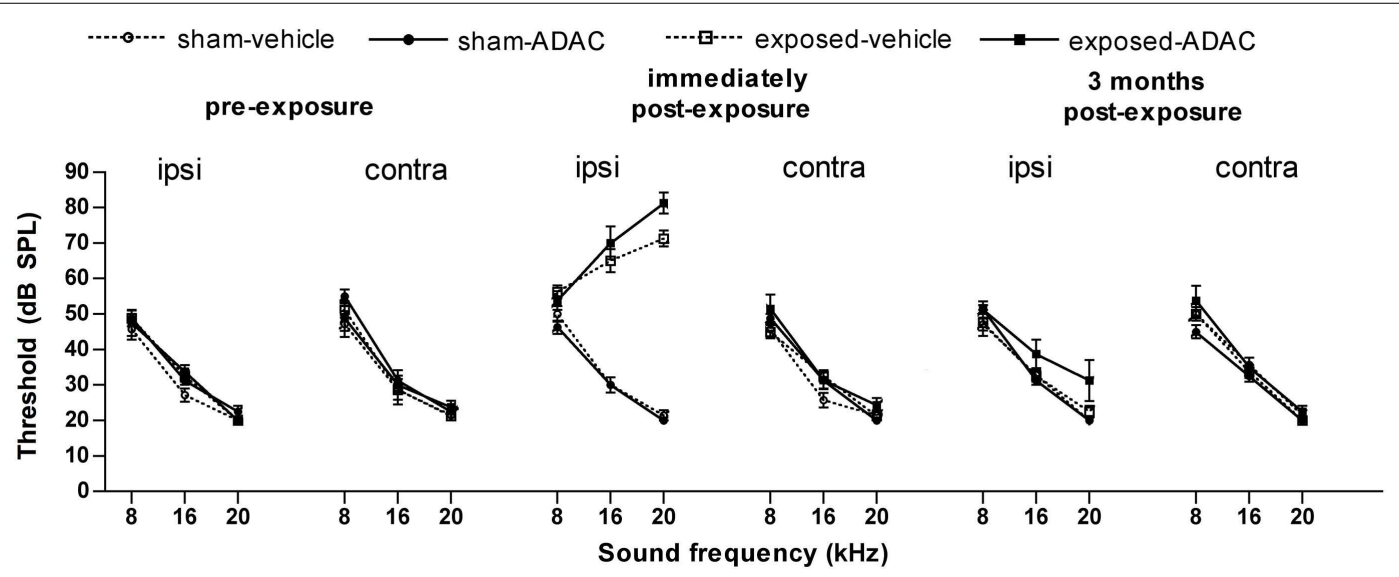

FIGURE 2 | Auditory brainstem-evoked response thresholds from another, similar experiment, in which measurements were made over $\mathbf{3}$ months following the acoustic trauma. Data are shown for the ipsilateral and contralateral ears of acoustic trauma-exposed and sham control animals before and after exposure, as a function of intensity in $\mathrm{dB}$ $\mathrm{SPL}$ and frequency in $\mathrm{kHz}$. Bars represent means $\pm 1 \mathrm{SE}$. The drug being tested in this study was ADAC, an adenosine amine congener (Zheng et al., unpublished observations). to a $32-\mathrm{kHz}$ tone. When the data for the individual animals was inspected, five out of eight of them met the criterion of at least three mean SR values that were lower than the sham control values, and therefore these animals (R3, 4, 5, 6, and 8) were considered to have tinnitus. Their individual data are shown in Figure 3.

After the confirmation of tinnitus in the pre-drug testing, the animals were injected with the saline vehicle every day while being tested for tinnitus. The results were similar following administration of the saline vehicle: the SR was significantly smaller in the acoustic trauma group in response to the $32-\mathrm{kHz}$ tones at higher stimulus intensities [interaction between group and intensity: $F(4,344.351)=3.44, P \leq 0.009$; data not shown] but not the $10 \mathrm{kHz}$ tones (for either group or the interaction between group and intensity). Although there was no significant group effect for the $\mathrm{BBN}$, there was a significant interaction between treatment and intensity $[F(4,334.85)=3.8, P \leq 0.005]$; however, this was due to the higher SRs for the noise-exposed animals at low stimulus intensities (data not shown).

\section{EFFECTS OF MEMANTINE}

When treated with $5 \mathrm{mg} / \mathrm{kg}$ memantine, only two of the five animals that exhibited tinnitus in the pre-drug condition, continued to exhibit it, i.e., only two of the same five animals (R4 and 8) that had at least three SR means lower than the sham control condition, continued to fulfill that criterion. This was a significant decrease in the proportion of animals with tinnitus, which had initially exhibited evidence of tinnitus ( $z=2.74, P \leq 0.006$; see Figure 4 for the data for the individual animals).

In order to ensure that the loss of behavioral signs of tinnitus in these animals was not due to the tinnitus merely disappearing by itself over time, the animals were given a washout period during which no drug treatment took place. During this period, three of 
$32 \mathrm{kHz}$ Pre-drug Exposed R1

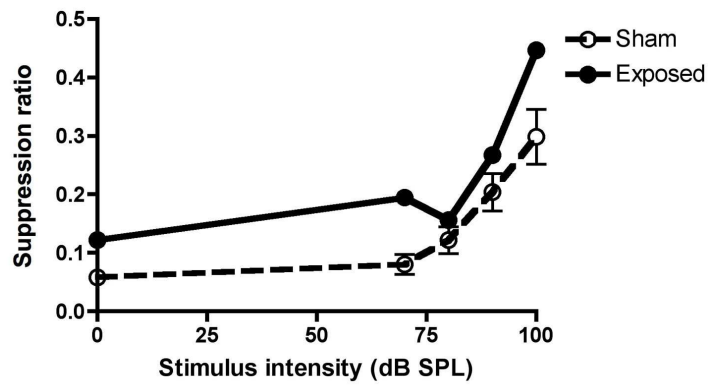

32 kHz Pre-drug exposed R2

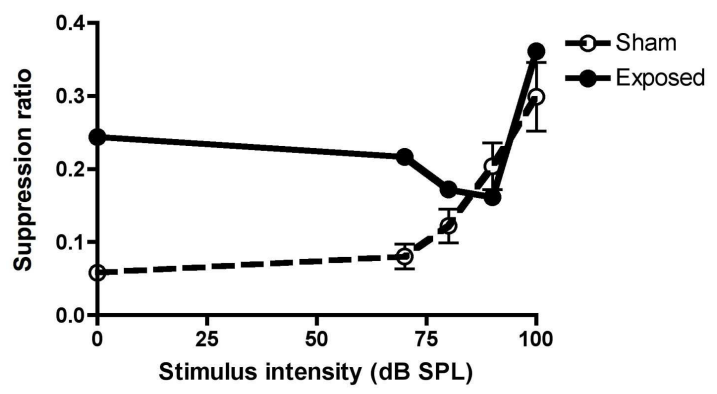

32 kHz Pre-drug exposed R3

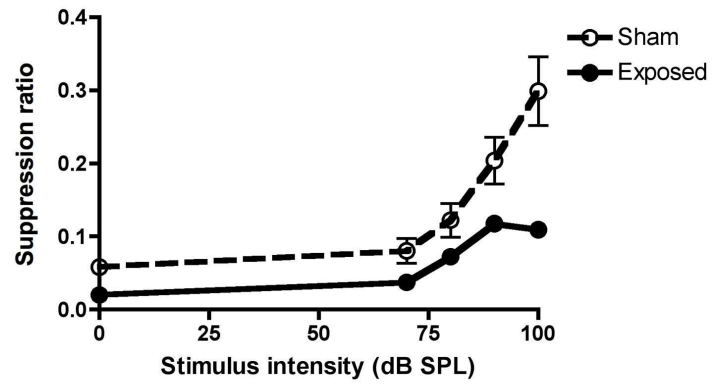

32 kHz Pre-drug exposed R4

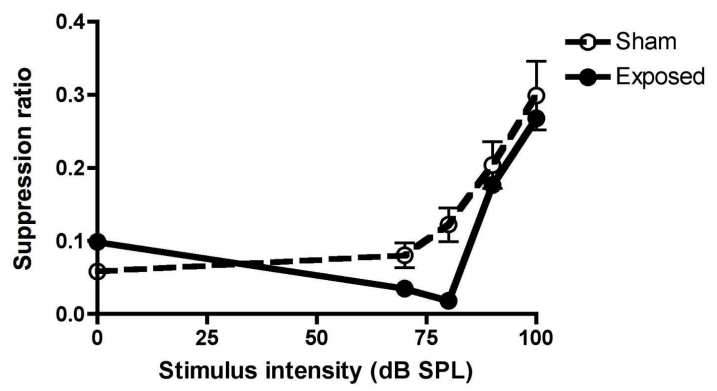

FIGURE 3 | Suppression ratios for the individual acoustic trauma-exposed animals plotted against the mean SRs of the sham control animals before any drug or vehicle administration, as a

the five animals which exhibited tinnitus in the pre-drug condition, continued to exhibit it (R3, 4, and 8) and this proportion was not significantly different from the pre-drug condition (see Figure 5 for the individual data).
$32 \mathrm{kHz}$ Pre-drug exposed R5

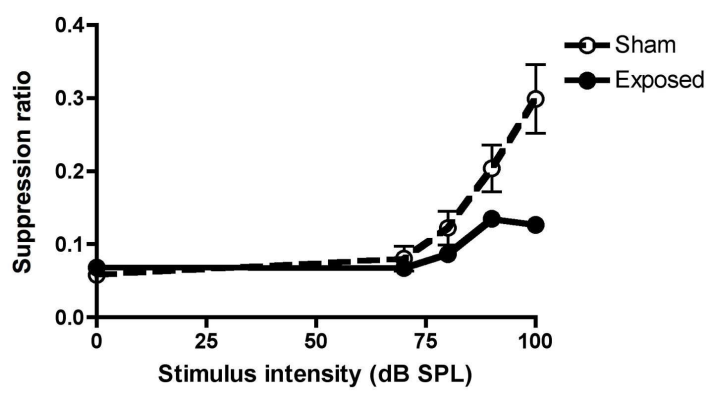

32 kHz Pre-drug exposed R6

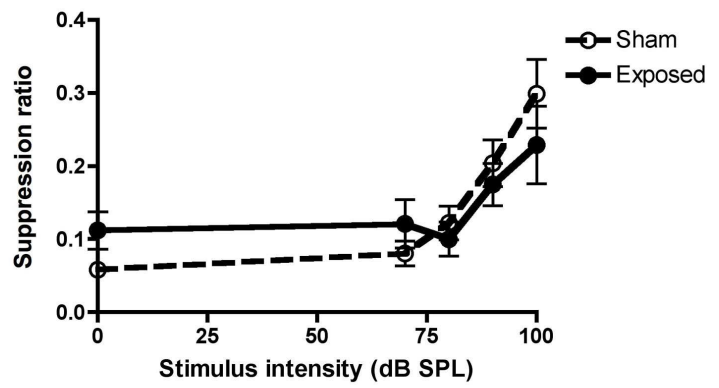

$32 \mathrm{kHz}$ Pre-drug exposed R7

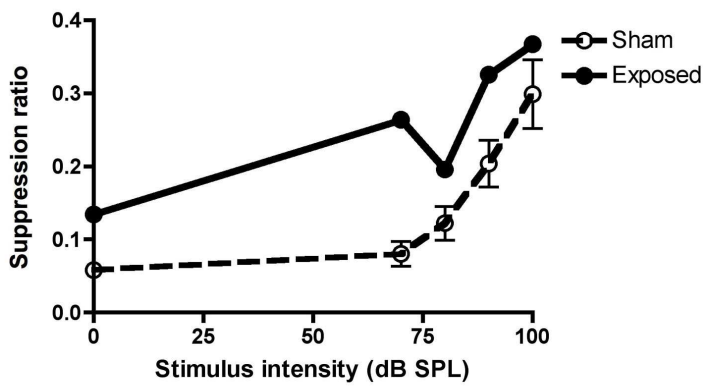

32 kHz Pre-drug exposed R8

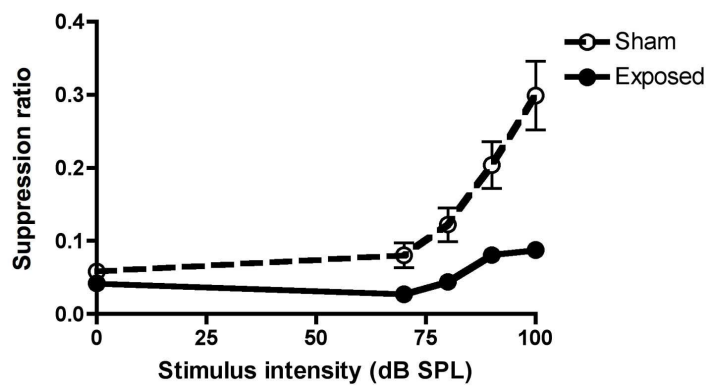

function of intensity in $\mathbf{d B}$ SPL to a $\mathbf{3 2} \mathbf{~ k H z}$ stimulus. R1-8 represent individual acoustic trauma-exposed animals. Symbols represent means $\pm 1 \mathrm{SE}$.

\section{DISCUSSION}

Consistent with our previous studies (Zheng et al., 2011a, 2012), we found that an acoustic trauma stimulus consisting of a $16 \mathrm{kHz}$ pure tone, at $110 \mathrm{~dB}$ SPL, delivered unilaterally for $1 \mathrm{~h}$, 
$32 \mathrm{kHz}$ Memantine exposed R1

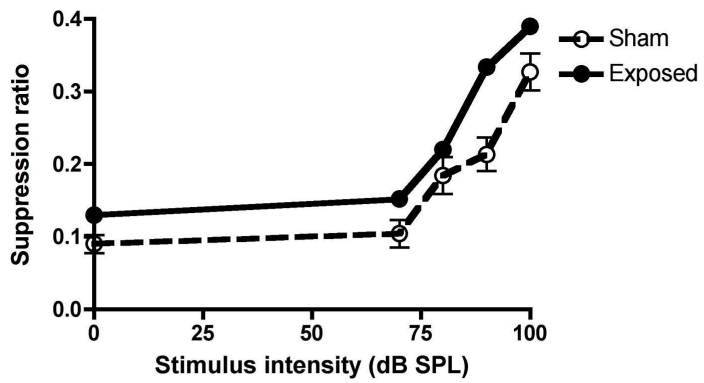

$32 \mathrm{kHz}$ Memantine exposed R2

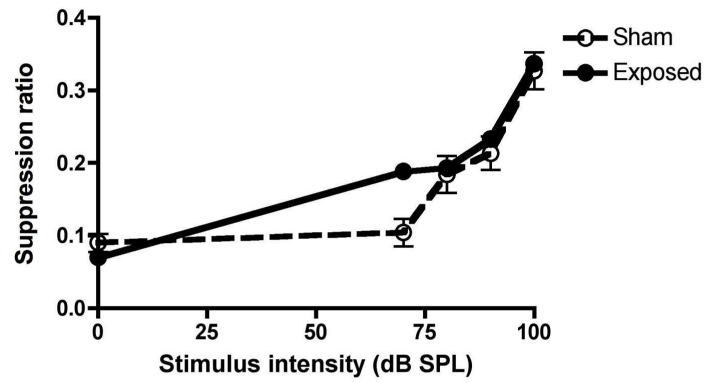

$32 \mathrm{kHz}$ Memantine exposed R3

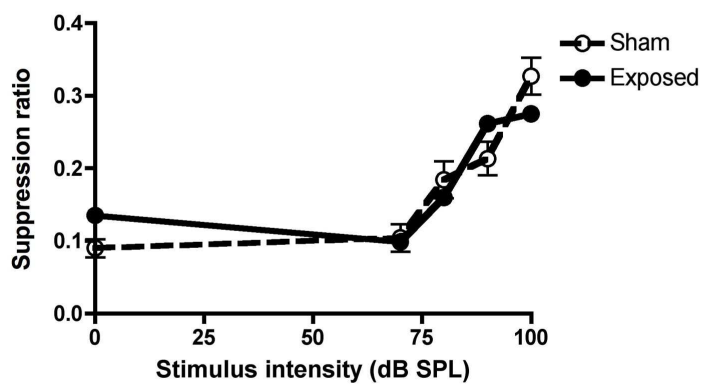

$32 \mathrm{kHz}$ Memantine exposed R4

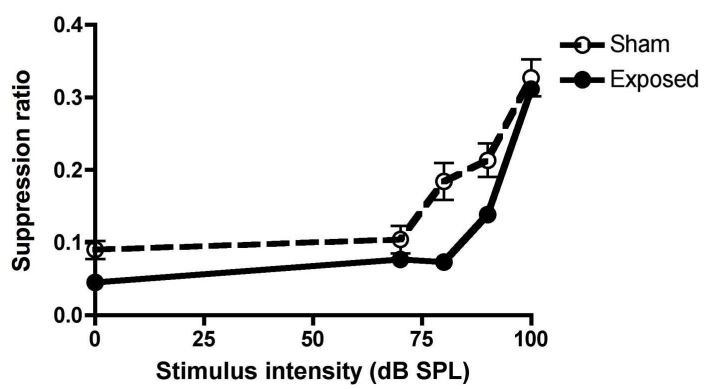

FIGURE 4 | Suppression ratios for the individual acoustic trauma-exposed animals plotted against the mean SRs of the sham control animals following the $5 \mathrm{mg} / \mathrm{kg}$ s.c. memantine administration, as

produced a large and significant increase in the ABR threshold in the affected ear. However, in contrast to our previous studies, this shift occurred only for tones at 16 and $20 \mathrm{kHz}$ and not for $8 \mathrm{kHz}$. We also observed a significant frequency specific
$32 \mathrm{kHz}$ Memantine exposed R5

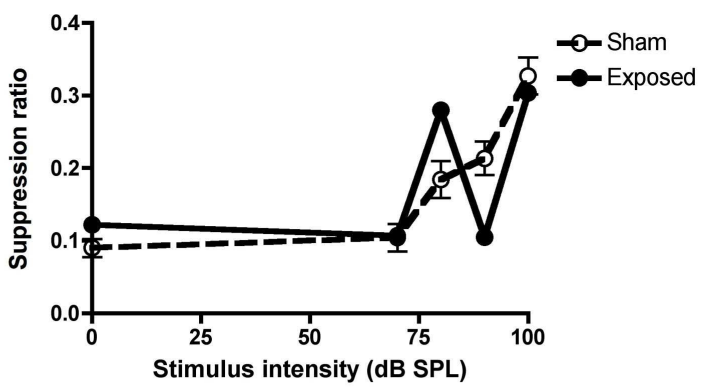

$32 \mathrm{kHz}$ Memantine exposed R6

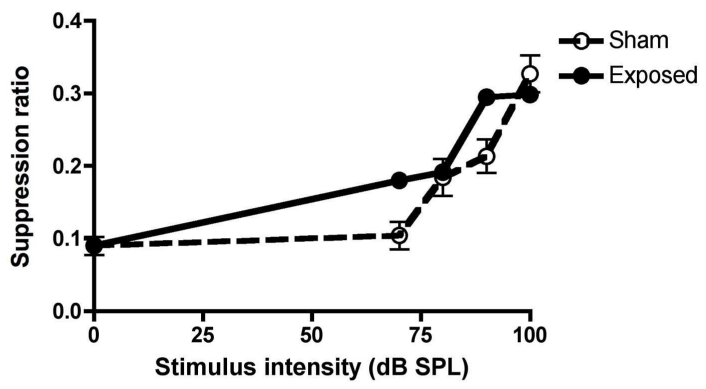

$32 \mathrm{kHz}$ Memantine exposed R7

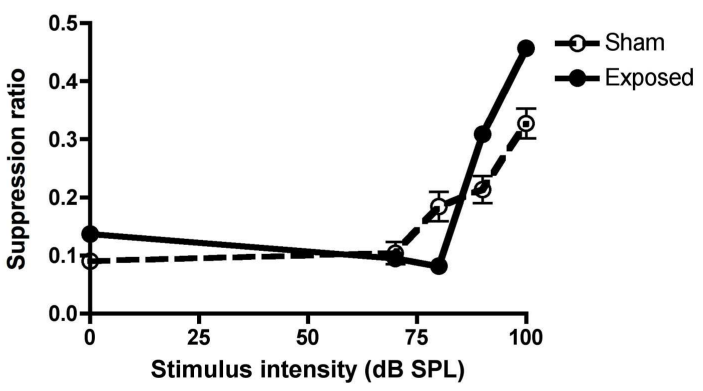

$32 \mathrm{kHz}$ Memantine exposed R8

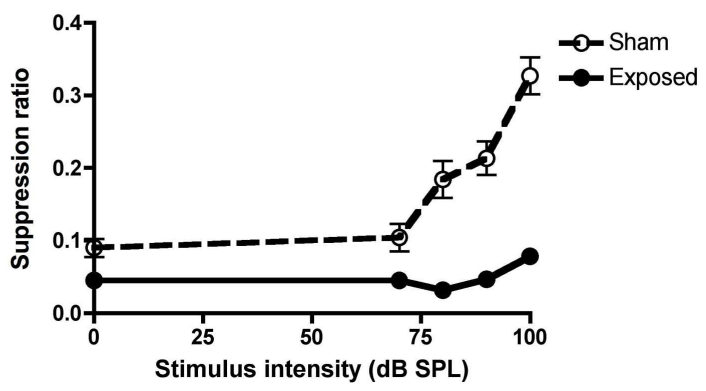

a function of intensity in $\mathrm{dB}$ SPL to a $32 \mathrm{kHz}$ stimulus. $\mathrm{R} 1-8$ represent individual acoustic trauma-exposed animals. Symbols represent means $\pm 1 \mathrm{SE}$

decrease in the average SR in a conditioned lick suppression paradigm, in response to $32 \mathrm{kHz}$ tones but not in response to $10 \mathrm{kHz}$ tones or BBN. Five out of eight animals tested fulfilled the criterion of at least three mean SR values in response to 
$32 \mathrm{kHz}$ Washout exposed R1

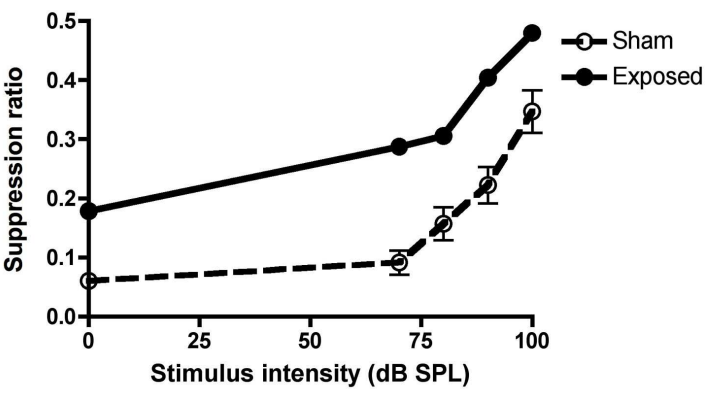

$32 \mathrm{kHz}$ Washout exposed R2

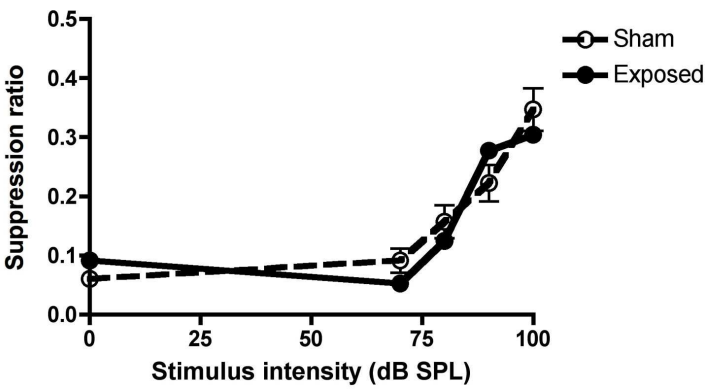

32 kHz Washout exposed R3

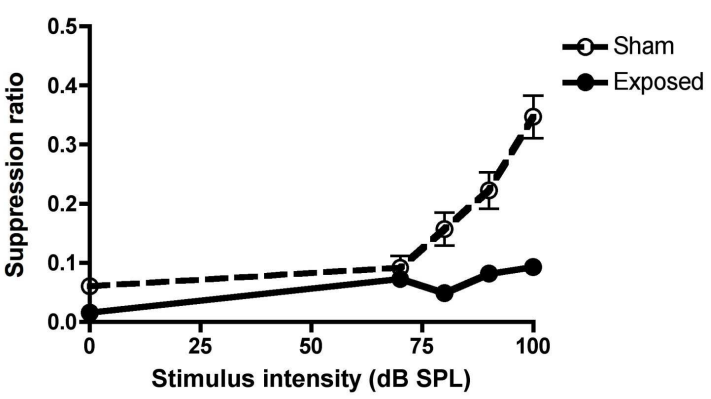

$32 \mathrm{kHz}$ Washout exposed R4

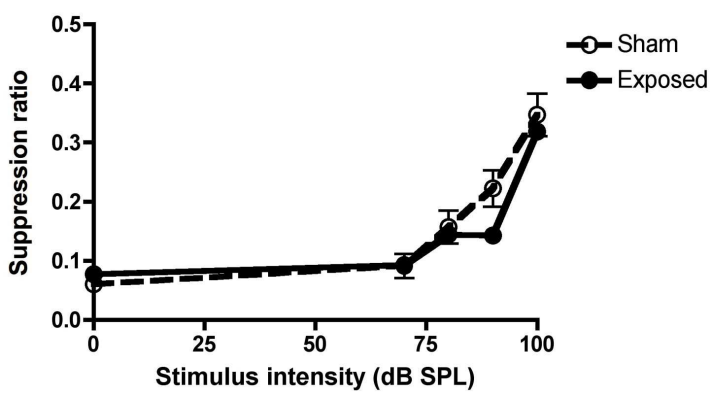

FIGURE 5 | Suppression ratios for the individual acoustic trauma-exposed animals plotted against the mean SRs of the sham control animals following the washout of the $5 \mathrm{mg} / \mathrm{kg}$ s.c. memantine
$32 \mathrm{kHz}$ Washout exposed R5

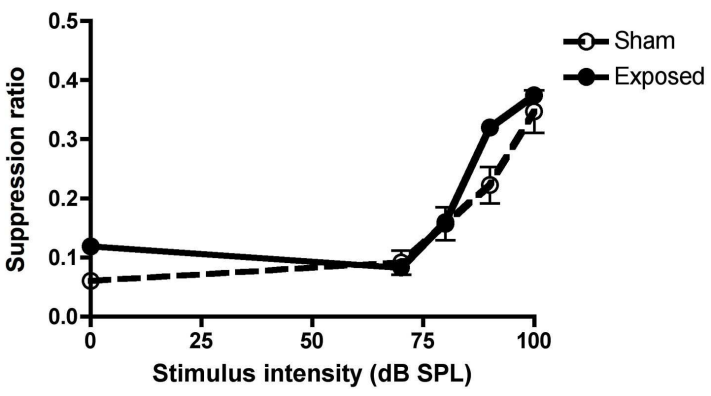

$32 \mathrm{kHz}$ Washout exposed R6

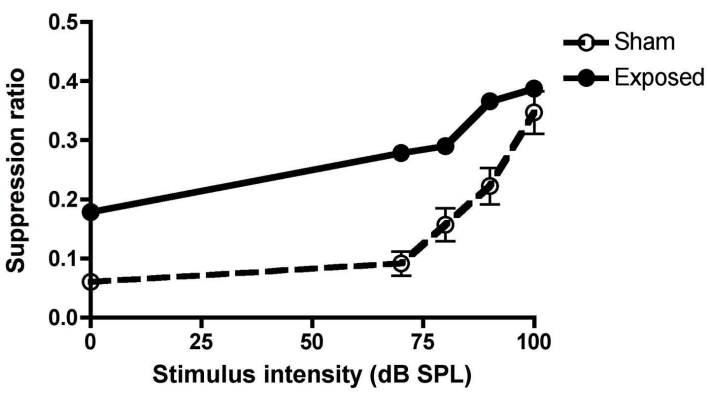

$32 \mathrm{kHz}$ Washout exposed R7

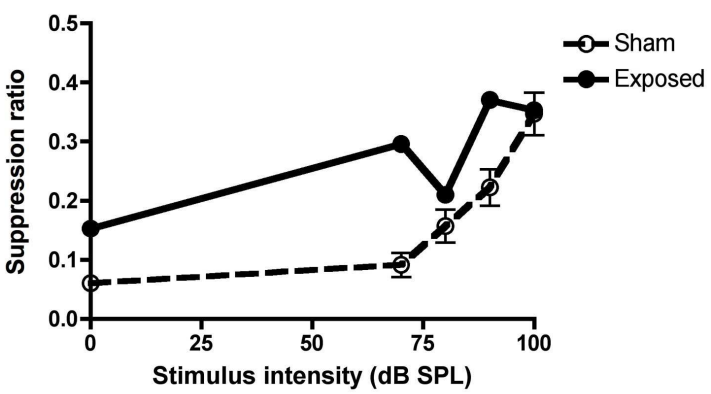

$32 \mathrm{kHz}$ Washout exposed R8

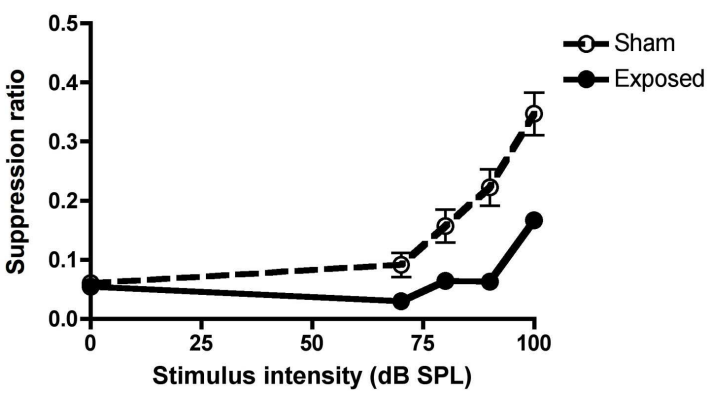

administration, as a function of intensity in $\mathrm{dB} S P L$ to a $32 \mathrm{kHz}$ stimulus. R1-8 represent individual acoustic trauma-exposed animals. Symbols represent means \pm 1 SE.
$32 \mathrm{kHz}$ tones being less than in the sham control condition and therefore these five animals were considered to exhibit evidence of tinnitus. In contrast to our previous studies (Zheng et al., 2011a, 2012), we found no change in the SR discrimination curve at $20 \mathrm{kHz}$. Memantine, at a dose of $5 \mathrm{mg} / \mathrm{kg}$ s.c, significantly reduced the number of animals exhibiting tinnitus from 
five to two, which then increased to $3 / 5$ during the washout period.

It is unclear to us why the same acoustic trauma produced less severe hearing loss at a lower frequency range (i.e., $8 \mathrm{kHz}$ ) when compared with our previous studies (Zheng et al., 2011a, 2012). It could be due to slight variations in the position of the speculum inserted in the external auditory canal. Nonetheless, this degree of damage was enough to produce the behavioral signs of tinnitus in 5/8 exposed animals. It is interesting to note that the frequency specific downward shift in the SR curve was at $32 \mathrm{kHz}$ rather than the $20 \mathrm{kHz}$ observed previously (Zheng et al., 2011a, 2012). Following noise trauma-induced hearing loss, the auditory cortex undergoes reorganization and neurons in the hearing loss frequency region begin to respond to frequencies of the neighboring neurons (Roberts et al., 2010). Therefore, with no ABR threshold changes at $8 \mathrm{kHz}$ in the present study, it is possible that the hearing loss was more pronounced at higher frequencies (i.e., $>20 \mathrm{kHz}$ as tested), which resulted in a perception of tinnitus at frequencies higher than $20 \mathrm{kHz}$. Nonetheless, we observed a clear reduction in the SRs in 5/8 acoustic trauma-exposed animals responding specifically to $32 \mathrm{kHz}$ tones.

It is difficult, if not impossible, to completely exclude the possibility that some aspect of the change in SR that we used to quantify tinnitus-related behavior, might be due to hearing loss caused by the acoustic trauma. We did not test long-term ABR thresholds in these animals at the end of this experiment. However, in a similar experiment involving eight sham and eight acoustic trauma-exposed rats, employing exactly the same acoustic trauma stimulus, we found no significant differences in hearing threshold at 3 months post-exposure, despite large and significant elevations of hearing threshold in the ipsilateral, exposed ear immediately after the noise trauma (Figure 2; Zheng et al., unpublished observations). This result suggests that, using our acoustic trauma stimulus, ABR thresholds gradually return toward normal over time. Since these animals exhibited behavioral evidence of confirmed tinnitus for about 2 months before the memantine treatment, this makes it less likely that the SR changes that we observed during the memantine treatment period were due simply to hearing loss.

Following the $5 \mathrm{mg} / \mathrm{kg}$ memantine treatment $1 \mathrm{~h}$ before each tinnitus testing session, we observed a significant attenuation of the shift in the SR curve at $32 \mathrm{kHz}$ in $3 / 5$ animals that exhibited tinnitus in the pre-drug condition. This suggests that the memantine significantly reduced tinnitus in the acoustic traumaexposed animals that developed tinnitus. This is the first evidence that systemic administration of memantine after the confirmation of tinnitus can effectively reduce tinnitus in an animal model. Lobarinas et al. (2006) reported that it had no significant effect on salicylate- and quinine-induced tinnitus in rats, or on the increase in cortical auditory-evoked potentials caused by salicylate (see also Lobarinas et al., 2008). However, drug- and noise-induced tinnitus are not necessarily comparable in terms of their mechanisms or response to drug treatments (see Eggermont and Roberts, 2004; Eggermont, 2005; Kaltenbach, 2006; Roberts et al., 2010 for reviews). Furthermore, Lobarinas et al. used lower doses (1.5 and $3 \mathrm{mg} / \mathrm{kg} /$ day) of memantine than we used in the current study $(5 \mathrm{mg} / \mathrm{kg})$. We did not conduct a dose-response analysis in this case because of the expense of memantine. Using the dose adjustment calculation employed by the FDA to calculate human equivalent doses (Reagan-Shaw et al., 2007), our dose of $5 \mathrm{mg} / \mathrm{kg}$ is approximately equivalent to $56.83 \mathrm{mg}$ /day for a $70 \mathrm{~kg}$ human adult. In the only published clinical trial, Figueiredo et al. (2008) found no significant effect of $20 \mathrm{mg}$ memantine per day on patients' tinnitus compared to placebo. It is conceivable that higher doses could have had a greater effect on the patients' tinnitus. However, adverse side effects may also have developed with higher doses.

Despite the previous evidence that memantine has little effect on tinnitus, Guitton and Dudai (2007) reported that acoustic trauma-induced tinnitus could be prevented by cochlear application of the NMDA receptor polyamine site antagonist, ifenprodil. However, this study showed that ifenprodil was only effective when administered within the first 4 days of noise trauma but not after tinnitus was "consolidated." In the present study, we showed that memantine was effective in reversing confirmed tinnitus in $3 / 5$ animals at least 4 weeks after the acoustic trauma. The main difference between our study and Guitton and Dudai's is the drug administration route. It is well accepted that although tinnitus can be initiated by noise trauma-induced hair cell damage, once it is established it is generated by hyperactive neurons in the brain (Roberts et al., 2010). It is possible that at an early stage, blocking NMDA receptors in the cochlea is enough to prevent the further development of tinnitus; once long-term tinnitus has developed, blockade of NMDA receptors in the central nervous system is necessary. Some beneficial effect on tinnitus patients has also been reported with neramexane, which is also an NMDA receptor antagonist with additional actions at the $\alpha_{9} \alpha_{10}$ nicotinic acetylcholine and 5- $\mathrm{HT}_{3}$ receptors (Suckfull et al., 2011). This clinical trial, along with the current evidence, suggests that memantine and similar drugs may be worthy of further consideration for the treatment of tinnitus.

In order to test the reversibility of memantine's effects on tinnitus, we evaluated the animals again during a washout period. Following the washout period, the SRs for the $32 \mathrm{kHz}$ stimulus were again lower than the sham control condition in $3 / 5$ animals. This proportion was not statistically different from that of the pre-drug condition and therefore provided some evidence that the tinnitus had returned in these animals following the washout of memantine. However, the result would have been more convincing if all five animals had again exhibited evidence of tinnitus, as they did in the pre-drug condition. The fact that this did not happen could be interpreted as a long-lasting effect of the drug on tinnitus, or could have been due to the washout period not being sufficiently long for the drug to be entirely eliminated, or merely because the tinnitus had disappeared over time by itself. It is very unlikely that the drug had not been entirely eliminated from the system given that the washout period lasted for 15 days; the half-life of a single dose of memantine in humans is about $65 \mathrm{~h}$ and the pharmacokinetics are very similar following multiple-doses for 14 days (Liu et al., 2008). It is also unlikely that tinnitus had disappeared by itself, because tinnitus had been confirmed in these animals in two separate testing periods, the pre-drug and the vehicle conditions. In other words, these animals had confirmed tinnitus for about 2 months before the memantine treatment. In our previous experiments, the longest time point when the exposed animals had confirmed tinnitus was at 10 months after the acoustic trauma (Zheng et al., 2011a), therefore, it seems unlikely that tinnitus 
would disappear in these animals after 2 months. However, we cannot exclude the possibility that the severity of the tinnitus fluctuated over time and the present study was not designed to investigate the longitude of memantine's effect on tinnitus. Further studies are required to explore this issue.

It has been reported that NMDA receptor antagonists, such as memantine, can impair associative learning (Schugens et al., 1997; Zajaczkowski et al., 1997). Therefore, it could be argued that the lack of difference in the frequency discrimination curve between the sham and exposed animals following memantine treatment was due to impaired associative learning produced by memantine. However, if this was the case, the animals would not have been able to produce lick suppression in response to all of the stimulus intensities tested and the frequency discrimination curves would be similar and shifted close to 0.5 across the stimulus intensity range. Moreover, the sham animals also received the same dose of memantine as the exposed animals. If memantine impaired the

\section{REFERENCES}

Agresti, A. (2007). Introduction to Categorical Data Analysis. New Jersey: Wiley.

Bauer, C. A., and Brozoski, T. J. (2001). Assessing tinnitus and prospective tinnitus therapeutics using a psychophysical animal model. J. Assoc. Res. Otolaryngol. 2, 54-64.

Brozoski, T. J., Spires, T. J. D., and Bauer, C. A. (2007). Vigabatrin, a GABA transaminase inhibitor, reversibly eliminates tinnitus in an animal model. J. Assoc. Res. Otolarnygol. 8, 105-118.

Darlington, C. L., and Smith, P. F. (2007). Drug treatments for tinnitus. Prog. Brain Res. 166, 249-262.

Dong, S., Mulders, W. H., Rodger, J., Woo, S., and Robertson, D. (2010). Acoustic trauma evokes hyperactivity and changes in gene expression in guinea-pig auditory brainstem. Eur. J. Neurosci. 31, 1616-1628.

Eggermont, J. J. (2005). Tinnitus: neurobiological substrates. Drug Discov. Today 10, 1283-1290.

Eggermont, J. J., and Roberts, L. E. (2004). The neuroscience of tinnitus. Trends Neurosci. 27, 676-682.

Elgoyhen, A. B., and Langguth, B. (2011). "Pharmacological approaches to tinnitus treatment," in Textbook of Tinnitus, eds A. R. Moller, B. Langguth, D. DeRidder, and T. Kleinjung (Heidelberg: Springer), 625-637.

Figueiredo, R. R., Langguth, B., Mello de Oliveira, P., and Aparecida de Azvedo, A. (2008). Tinnitus treatment with memantine. Otolaryngol. Head Neck Surg. 138, 492-496.

Guitton, M. J., Caston, J., Ruel, J., Johnson, R. M., Pujol, R., and Puel, J.
L. (2003). Salicylate induces tinnitus through activation of cochlear NMDA receptors. J. Neurosci. 23, 3944-3952.

Guitton, M. J., and Dudai, Y. (2007). Blockade of cochlear NMDA receptors prevents long-term tinnitus during a brief consolidation window after acoustic trauma. Neural Plast. 80904.

Gurka, M. J., and Edwards, L. J. (2011). "Mixed models," in Essential Statistical Methods for Medical Statistics, eds C. R. Rao, J. P. Miller, and D. C. Rao (Amsterdam: Elsevier), 146-173.

Heffner, H. E., and Harrington, I. A. (2002). Tinnitus in hamsters following exposure to intense sound. Hear. Res. 170, 83-95.

Kaltenbach, J. A. (2006). The dorsal cochlear nucleus as a participant in the auditory, attentional and emotional components of tinnitus. Hear. Res. 216, 224-234.

Kutner, M. H., Nachtsheim, C. J., Neter, J., and Li, W. (2005). Applied Linear Statistical Models. Boston: McGrawHill Irwin.

Leaver, A. M., Renier, L., Chevillet, M. A., Morgan, S., Kim, H. J., and Rauschecker, J. P. (2011). Dysregulation of limbic and auditory networks in tinnitus. Neuron 69, 33-43.

Lin, X. (1997). Action potentials and underlying voltage-dependent currents studied in cultured spiral ganglion neurons of the postnatal gerbil. Hear. Res. 108, 157-179.

Liu, M. Y., Meng, S. N., Wu, H. Z., Wang, S., and Wei, M. J. (2008). Pharmacokinetics of single-dose and multipledose memantine in healthy Chinese volunteers using an analytic method of liquid chromatography-tandem mass spectrometry. Clin. Ther. 30, 641-653.

conditioned lick suppression response, there should have been a significant change in the frequency discrimination curve before and after the drug treatment in the sham animals. However, this was not the case.

In conclusion, this study provides the first evidence that memantine may reduce tinnitus in rats caused by acoustic trauma. It may therefore be worth further investigating the potential therapeutic effects of memantine and similar drugs in the treatment of human tinnitus.

\section{ACKNOWLEDGMENTS}

This research was supported by grants from the Jean Cathie Estate to Paul F. Smith, Yiwen Zheng, and Cynthia L. Darlington. Emily McNamara and Lucy Stiles were Assistant Research Fellows supported by the Jean Cathie Estate. Yiwen Zheng was supported by a New Zealand Health Research Council Sir Charles Hercus Fellowship.

Lobarinas, E., Sun, W., Stolzberg, D. Jianzhong, L., and Salvi, R. (2008). Human brain imaging of tinnitus and animal models. Semin. Hear. 24, 333-349.

Lobarinas, E., Yang, G., Sun, W., Ding, D., Mirza, N., Dalby-Brown, W., Hilczmayer, E., Fitzgerald, S., Zhang, L., and Salvi, R. (2006). Salicylateand quinine-induced tinnitus and effects of memantine. Acta Otolaryngol. Suppl. 126, 13-19.

Middleton, J. W., Kiritani, T., Pedersen, C., Turner, J. G., Shepherd, G. M., and Tzounopoulos, T. (2011). Mice with behavioral evidence of tinnitus exhibit dorsal cochlear nucleus hyperactivity because of decreased GABAergic inhibition. Proc. Natl. Acad. Sci. U.S.A. 108, 7601-7606.

Moller, A. R. (2000). Similarities between severe tinnitus and chronic pain. J. Am. Acad. Audiol. 11, 115-124.

Mulders, W. H., and Robertson, D. (2011). Progressive centralization of midbrain hyperactivity after acoustic trauma. Neuroscience 192, 753-760.

Norusis, M. J. (2010). PASW18 Statistics 18 Advanced Statistical Procedures Companion. New Jersey: Prentice Hall.

Olivares, D., Deshpande, V. K., Shi, Y., Lahiri, D. K., Greig, N. H., Rogers, J. T., and Huang, X. (2012). NMethyl D-Aspartate (NMDA) receptor antagonists and memantine treatment for Alzheimer's disease, vascular dementia, and Parkinson's disease. Curr. Alzheimer Res. 9, 746-758.

Rauschecker, J. P., Leaver, A. M., and Mühlau, M. (2010). Tuning out the noise: limbic-auditory interactions in tinnitus. Neuron 66, 819-826.
Reagan-Shaw, S., Nihal, M., and Ahmad, N. (2007). Dose translation from animal to human studies revisited. FASEB J. 22, 659-661.

Roberts, L. E., Eggermont, J. J., Caspary, D. M., Shore, S. E., Melcher, J. R., and Kaltenbach, J. A. (2010). Ringing ears: the neuroscience of tinnitus. J. Neurosci. 30, 14972-14979.

Schugens, M. M., Egerter, R., Daum, I., Schepelmann, K., Klockgether, T., and Löschmann, P. A. (1997). The NMDA antagonist memantine impairs classical eyeblink conditioning in humans. Neurosci. Lett. 224, 57-60.

Shargorodsky, J., Curhan, G. C., and Farwell, W. R. (2010). Prevalence and characteristics of tinnitus among US adults. Am. J. Med. 123, 711-718.

Sprent, P., and Smeeton, N. C. (2007). Applied Nonparametric Statistical Methods. Boca Raton: Chapman and Hall/CRC.

Suckfull, M., Althaus, M., Ellers-Lenz, B., Gebauer, A., Gortelmeyer, R., Jastreboff, P. J., Moebius, H. J., Rosenberg, T., Russ, H., Wirth, Y., Krueger, H. (2011). A randomized, doubleblind, placebo-controlled clinical trial to evaluate the efficacy and safety of neramexane in patients with moderate to severe subjective tinnitus. BMC Ear Nose Throat Disord. 11, 1. doi:10.1186/1472-681511-1

Tan, J., Rüttiger, L., Panford-Walsh, R., Singer, W., Schulze, H., Kilian, S. B., Hadjab, S., Zimmermann, U., Köpschall, I., Rohbock, K., and Knipper, M. (2007). Tinnitus behavior and hearing function correlate with the reciprocal expression patterns of BDNF and Arg3.1/arc in auditory neurons following acoustic trauma. Neuroscience 145, 715-726. 
Vogler, D. P., Robertson, D., and Mulders, W. H. (2011). Hyperactivity in the ventral cochlear nucleus after cochlear trauma. J. Neurosci. 31, 6639-6645.

Wang, H., Brozoski, T. J., and Caspary, D. M. (2011). Inhibitory neurotransmission in animal models of tinnitus: maladaptive plasticity. Hear Res. 9, 111-117.

Zajaczkowski, W., Frankiewicz, T., Parsons, C. G., and Danysz, W. (1997). Uncompetitive NMDA receptor antagonists attenuate NMDA-induced impairment of passive avoidance learning and LTP. Neuropharmacology 36, 961-971.

Zheng, Y., Hamilton, E., Stiles, L., McNamara, E., de Waele, C., Smith,
P.F. and Darlington, C. L. (2011a). Acoustic trauma that can cause chronic tinnitus impairs impulsive control but not performance accuracy in the 5-choice serial reaction time task in rats. Neuroscience 180, 75-84.

Zheng, Y., Hamilton, E., Begum, S., Smith, P. F., and Darlington, C. L. (2011b). The effects of acoustic trauma that can cause tinnitus on spatial performance in rats. Neuroscience 186, 48-56.

Zheng, Y., Hamilton, E., McNamara, E., Smith, P. F., and Darlington, C. L. (2011c). The effects of chronic tinnitus caused by acoustic trauma on social behaviour and anxiety in rats. Neuroscience 193, 143-153.

Zheng, Y., Vagal, S., McNamara, E. Darlington, C. L., and Smith, P. F. (2012). A dose-response analysis of the effects of L-baclofen on chronic tinnitus caused by acoustic trauma in rats. Neuropharmacology 62, 940-946.

Conflict of Interest Statement: The authors declare that the research was conducted in the absence of any commercial or financial relationships that could be construed as a potential conflict of interest.

Received: 11 May 2012; accepted: 25 July 2012; published online: 15 August 2012.
Citation: Zheng $Y$, McNamara E, Stiles L, Darlington CL and Smith PF (2012) Evidence that memantine reduces chronic tinnitus caused by acoustic trauma in rats. Front. Neur. 3:127. doi: 10.3389/fneur.2012.00127

This article was submitted to Frontiers in Neuro-otology, a specialty of Frontiers in Neurology.

Copyright (c) 2012 Zheng, McNamara, Stiles, Darlington and Smith. This is an open-access article distributed under the terms of the Creative Commons Attribution License, which permits use, distribution and reproduction in other forums, provided the original authors and source are credited and subject to any copyright notices concerning any third-party graphics etc. 